\title{
Search for variations in circular-polarization spectra of the magnetic white dwarf LP 790-29*
}

\author{
S. Jordan ${ }^{1,2,3}$ and S. Friedrich ${ }^{1,4}$ \\ 1 Institut für Theoretische Physik und Astrophysik, Universität Kiel, 24098 Kiel, Germany \\ 2 Universitäts-Sternwarte, Geismarlandstraße 11, 37083 Göttingen, Germany, \\ 3 Institut für Astronomie und Astrophysik, Sand 1, 72076 Tübingen, Germany \\ e-mail: jordan@astro.uni-tuebingen.de \\ 4 Astrophysikalisches Institut Potsdam, An der Sternwarte 16, 14482 Potsdam, Germany \\ e-mail: sfriedrich@aip.de
}

Received 21 September 2001 / Accepted 22 November 2001

\begin{abstract}
We present highly time resolved circular-polarization and flux spectra of the magnetic white dwarf LP 790-29 taken with the VLT UT1 in order to test the hypothesis that LP 790-29 is a fast rotator with a period of the order of seconds to minutes. Due to low time resolution of former observations this might have been overlooked - leading to the conclusion that LP 790-29 has a rotational period of over 100 years. The optical spectrum exhibits one prominent absorption feature with minima at about 4500, 4950, and $5350 \AA$, which are most likely $\mathrm{C}_{2}$ Swan-bands shifted by about $180 \AA$ in a magnetic field between $50 \mathrm{MG}$ and $200 \mathrm{MG}$. At the position of the absorption structures the degree of circular polarization varies between $-1 \%$ and $+1 \%$, whereas it amounts to +8 to $+10 \%$ in the blue and red continuum. With this very high degree of polarization LP 790-29 is very well suited to a search for short time variations, since a variation of several percent in the polarization can be expected for a magnetic field oblique to the rotational axis. From our analysis we conclude that variations on time scales from 50 to 2500 s must have amplitudes $\lesssim 0.7 \%$ in the continuum and $\lesssim 2 \%$ in the strongest absorption feature at $4950 \AA$. While no short-term variations could be found a careful comparison of our polarization data of LP 790-29 with those in the literatures indicates significant variations on time scales of decades with a possible period of about $24-28$ years.
\end{abstract}

Key words. stars: individual: LP 790-29 - stars: rotation - stars: white dwarfs - stars: magnetic fields

\section{Introduction}

LP 790-29 belongs to a small group of magnetic white dwarfs which were suspected to be polarimetrically constant (West 1989; Schmidt \& Norsworthy 1991) implying rotational periods of more than 100 years. Alternatively, the non-detection of variations in the polarization can also mean a rotationally symmetric magnetic field geometry or a rotational period too short $(P<10 \mathrm{~min}$, Schmidt \& Norsworthy 1991) to be time-resolved as yet. The shortest period of 12 min found in a (magnetic) white dwarf was measured in RE J0317-853 (Barstow et al. 1995; Burleigh et al. 1999) meaning either that angular momentum is conserved to a higher degree or that the star is the product of merging. Segretain et al. (1997) predicted rotational

Send offprint requests to: S. Jordan,

e-mail: jordan@astrophysik.uni-kiel.de

* Based on observations collected at the European Southern Observatory, Paranal, Chile (ESO Programme 65.H-0293). velocities of about $1000 \mathrm{~km} \mathrm{~s}^{-1}(P \approx 40 \mathrm{~s})$ from their models of merging white dwarfs.

If angular momentum is completely conserved during stellar evolution, rotational periods just above the breakup limit of a few seconds are even possible in the case of single white dwarfs. If any of the strongly magnetic white dwarfs really turns out to be an extremely fast rotator it may generate a significant anisotropic moment of inertia and thereby gravitational radiation measurable with space interferometers (Heyl 2000).

Since such a fast rotation cannot be excluded from previous observations we obtained highly time-resolved flux and circular-polarization spectra of LP 790-29, continuing our search for short rotational periods in white dwarfs. Friedrich \& Jordan (2001) have started such a search with a broad-band photometric study of the linear polarization in the famous magnetic white dwarf Grw $+70^{\circ} 8247$. So far, no indications for fast rotation could be found, which means that the angular momentum has been almost completely lost during stellar evolution. Recently, 
long-term variations in the polarization of GD 229 and G 240-7 have been observed, from which a rotational period of about 100 years can be deduced (Berdyugin \& Piirola 1999). The puzzling slow rotation of most white dwarf stars is usually explained by magnetic braking of the stellar core during the process of bipolar outflows that may produce the observed bipolar planetary nebulae (Blackman et al. 2001). Even without a magnetic field a third dredge-up can very efficiently transport angular momentum from the core of an AGB star to the envelope so that the final white dwarf essentially stops rotating $\left(v_{\text {rot }}=10^{-3} \mathrm{~km} \mathrm{~s}^{-1}\right.$, Driebe \& Blöcker 2001).

With its very strong wavelength dependence of the degree of circular polarization ( $-1 \%$ to $+10 \%)$ LP $790-29$ is best suited for such a study. If LP 790-29 has a magnetic field not exactly aligned to its rotational axis, a variation of several percent can be expected. In the extreme case of a rotational axis perpendicular to both the observer and the magnetic field axis the polarization in the continuum could vary between $0 \%$ and $20 \%$ in order to account for the mean observed value in the continuum of about $10 \%$. The only shortcoming of observing this particular object is that no reliable quantum mechanical calculations exist as yet for the $\mathrm{C}_{2}$ molecule features seen in LP 790-29, but simple estimations explain the shifts of the Swan bands by $500 \AA$ with magnetic fields strengths between 50 MG (Bues 1999) and 200 MG (Liebert et al. 1978; Schmidt et al. 1995); therefore flux and polarization cannot be compared to theoretical models in order to measure the Doppler broadening.

\section{Observation and data reduction}

Observations of LP 790-29 were performed on May 5, July 3 and 4, 2000, in service mode by ESO staff members with VLT FORS1 in standard resolution. Grism GRIS 150I+17 was used without an order sorting filter to cover the spectral range below $6500 \AA$ with a resolution of $5.4 \AA /$ pixel. A Wollaston prism and a quarter wave plate were inserted to obtain circular polarization spectra. The latter was rotated by $90^{\circ}$ after each consecutive exposure to account for errors in flatfielding, offsets from the retarder plate zero angle, and wavelength dependencies of the retardation of the quarter wave plate. Flat field, bias, and wavelength calibration exposures were taken with the same instrument configuration after each observing night. Sky spectra were taken from regions on the CCD adjacent to the observed stars and subtracted from the object spectra. One anonymous field star at a distance of $1^{\prime}$ to LP 790-29, assumed to be non-variable, was simultaneously observed, to check for observationally introduced periods and to obtain a measure for the accuracy of the polarization data. Exposure times were varied between $11 \mathrm{~s}$ and $83 \mathrm{~s}$, in order to avoid aliases in periodograms as a consequence of a regular observing scheme. In order to reduce read-out time and overhead of the CCD only a window of 650 pixel $\times 2048$ pixel was read out.

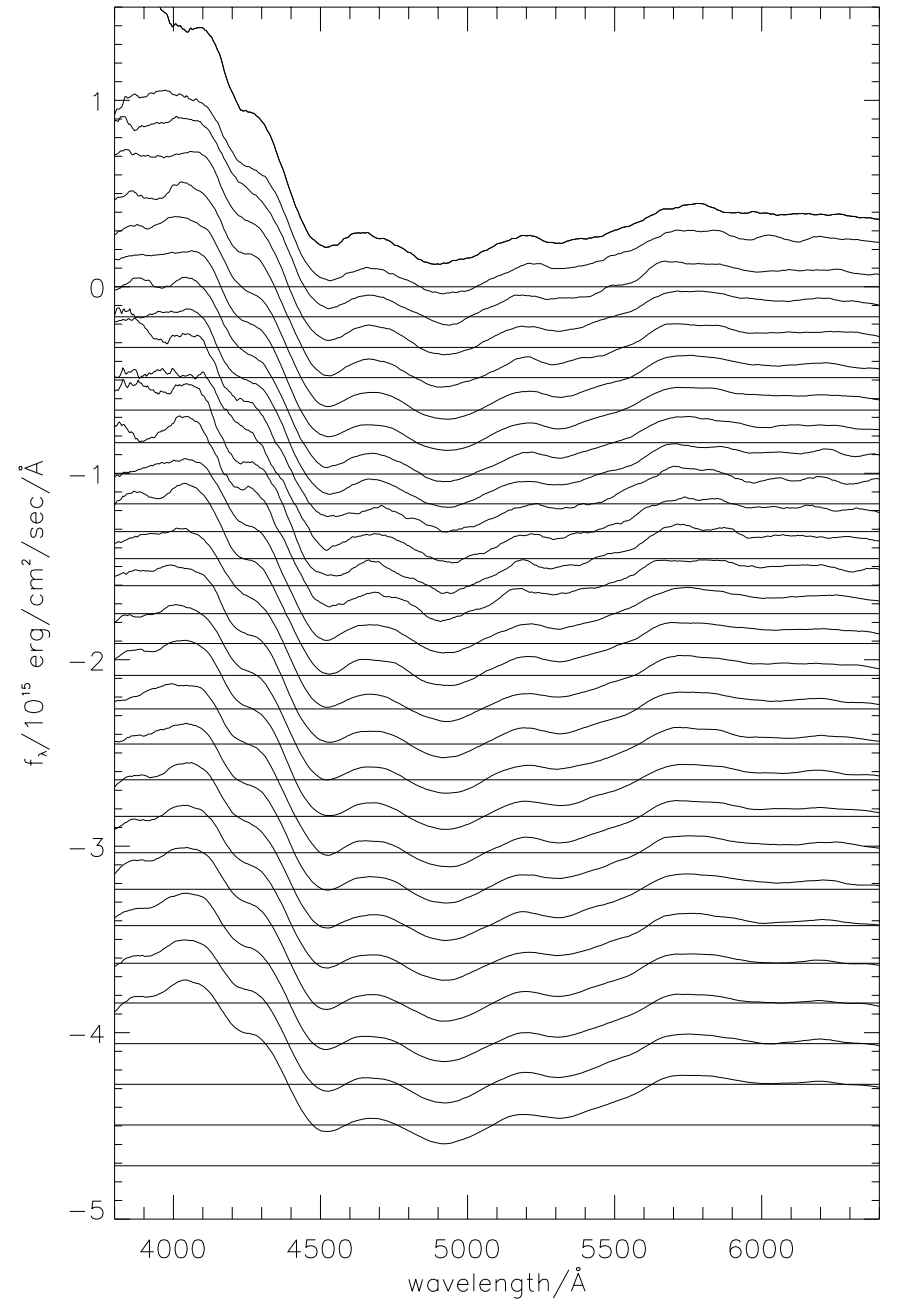

Fig. 1. Phase resolved and Savitzki-Golay filtered flux spectra of LP 790-29 of the July 3-4, 2000, observing run. The spectra are shifted proportional to the time past since the first observation (total time: $4800 \mathrm{~s}$ ). The zero point of the ordinate corresponds to the first (upper) exposure. For all observations a line is drawn to indicate the zero level.

Finally a flux standard (GD108) was observed for a rough flux calibration.

Data reduction was performed with standard IRAF routines. The circular polarization of an individual exposure was determined as the difference between the two object spectra divided by their sum. The final circular polarization (corrected for observationally introduced errors) was determined from two consecutive exposures whose retarder angle differed by $90^{\circ}$.

During the first observing run on May 5 LP 790-29 was observed 9 times with effective exposure times between 22 and $90 \mathrm{~s}$; two consecutive exposures with different retarder angles were used together to calculate the polarization. Probably due to the atmospheric conditions the measured flux of the comparison star was highly variable; it had a flux level up to a factor three lower than in the second period in July, where the star varied by less than $10 \%$. After 20 min the May observing run was cancelled. Due to the worse exposure the photon noise in the 


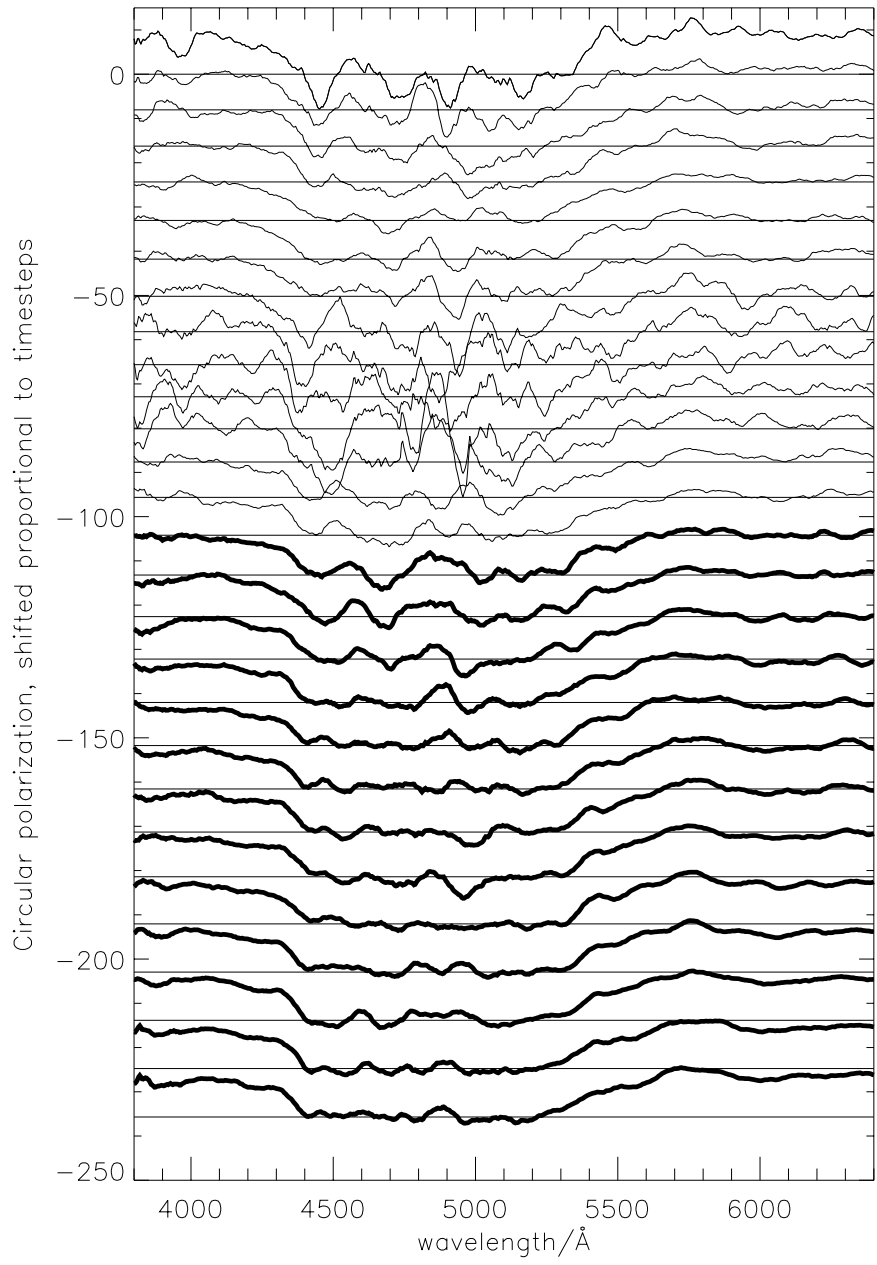

Fig. 2. Circular polarization of LP 790-29 for 26 phases of the second observation period covering a total of $4600 \mathrm{~s}$. The noise was reduced with a Savitzki-Golay filter of $300 \AA$ width and a 4th degree polynomial. The zero points of the degrees of polarization for all observations are shown as horizontal lines. Data taken with more than $100 \mathrm{~s}$ exposure time are marked with thick lines.

polarization had strongly increased so that we based our analysis almost entirely on the data from the July observations. In total we obtained only about half of the exposure time originally granted to this project, but if the polarization amplitude were a large fraction of the $10 \%$ expected for a perpendicular rotation this would be sufficient to measure a period. The effective exposure times for the 26 observations during the July run vary between 22 and $166 \mathrm{~s}$.

\section{Data analysis}

In Figs. 1 and 2 we have plotted the flux $f_{\lambda}$ and the circular polarization for all measurements of the second observing run. Our unsmoothed polarization data $(5.4 \AA /$ pixel $)$ have a mean noise level of about $\pm 6 \%$ - too large to directly search for variations in the data. Therefore, the observations shown in Fig. 2 had to be smoothed. Since we did not want to broaden potential structures in the

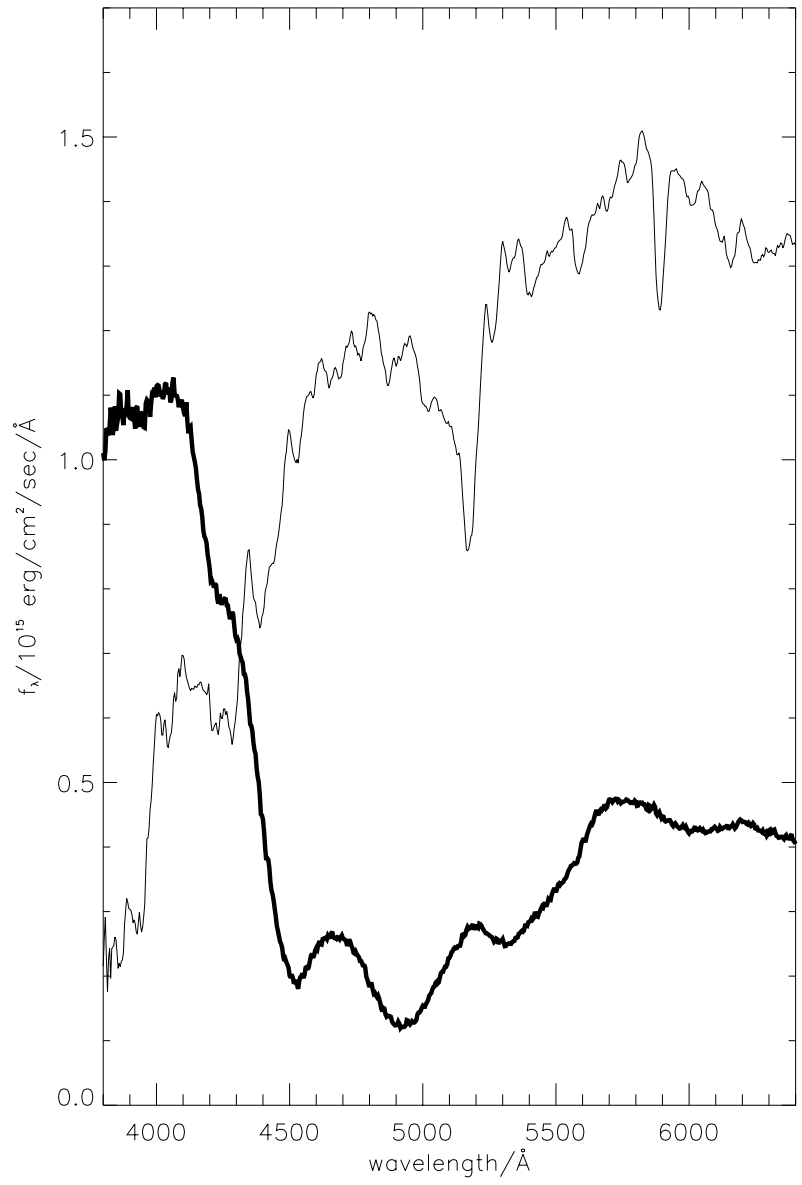

Fig. 3. Mean flux spectra of LP 790-29 and of the unpolarized comparison star.

polarization spectrum we used a Savitzki-Golay filter (300 $\AA$ width and a 4th degree polynomial; Press et al. 1992) which, compared to simple Gaussian or boxcar filters, preserves the height and width of narrow features, still considerably reducing the noise.

On first glance the polarization data seem to indicate large variations which, however, are mostly present in the observations taken with less than $100 \mathrm{~s}$ exposure time (Fig. 2). Without a careful inspection it is difficult to judge whether the variations in the polarization can be trusted or whether these changes are entirely due to the higher noise level which has only been partly reduced by the filtering.

For this reason we tried to obtain a more quantitative measure for the signal-to-noise ratio. We were following two different approaches: Firstly, variations of the measured degree of circular polarization in the comparison star should be pure noise. Therefore we determined the standard deviation $\sigma$ from zero for all observations of the calibration star. We found that $\sigma \cdot \sqrt{t_{\text {exposure }}}$ was nearly a constant so that the noise can approximately be described by Poisson statistics. We estimated $\sigma_{\mathrm{LP}}(\lambda)$ in LP 790-29 by multiplying $\sigma_{\text {comp }}(\lambda)$ with the square root of the flux ratio $\sqrt{f_{\lambda, \mathrm{comp}} / f_{\lambda, \mathrm{LP}}}$.

Secondly, we determined $\sigma_{\mathrm{LP}}(\lambda)$ without reference to the comparison star: For several wavelength intervals we 


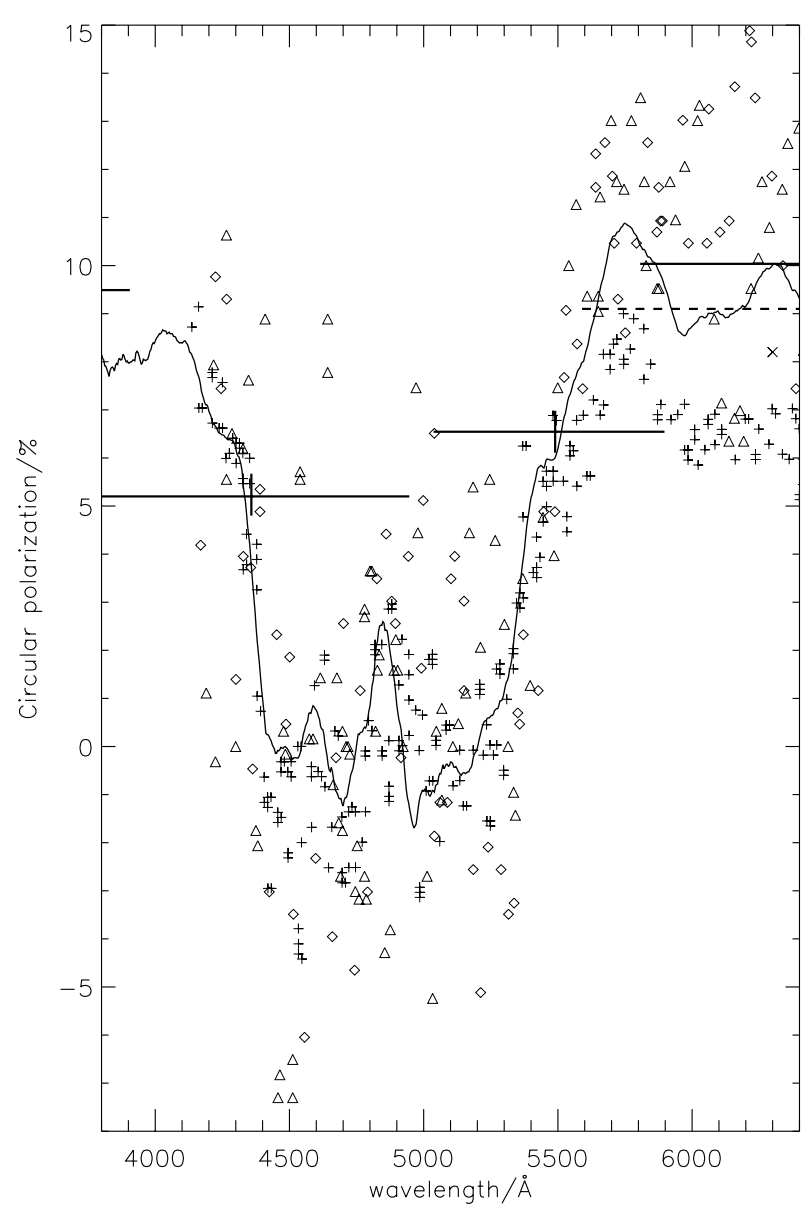

Fig. 4. Comparison of the mean of our VLT measurements of circular polarization (curve) to data from the literature: February 22, 1977 (triangles), and February 23, 1977 (diamonds), taken by Liebert et al. (1978), May 7-8, 1994, (plus signs) observed by Schmidt et al. (1995). For a clearer distinction these measurements were not connected. The broad-band data by West (1989), marked by error bars, were taken between January 1986, and September 1988, the measurement in the red by Robert \& Moffat (1989, February 23, 1987, "x" symbol) and by Beuermann \& Reinsch (2001; dashed line) taken on February 4, 2000.

used the standard deviation of the differences between the data and the Savitzki-Golay filtered observations as a measure for the noise. We concluded that the $\sigma_{\mathrm{LP}}(\lambda)$ estimated with the second method was about one third larger than with the first method, probably an indication for small non-Poisson contributions.

We used the larger value for a simulation: we added artificial Gaussian noise with the $\sigma_{\mathrm{LP}}(\lambda)$ estimated above to the mean polarization spectrum and compared the resulting synthetic spectra to the observation. In both data sets the variations are of the same order. We also could not find any significant differences between the May and July observation runs, and therefore we conclude that variations of more than $2 \%$ on time scales of minutes or months are unlikely.

\section{Period search}

Nevertheless we tried to search for periodicities using both the analysis of variance method by SchwarzenbergCzerny (1989) and the phase dispersion minimization algorithm by Stellingwerf (1978); no significant differences were found between the results of the two methods.

We searched for periods between 50 and $2500 \mathrm{~s}$ in (a) the mean polarization at the wavelengths of the strong absorption structure between 4300 and $5400 \AA$, (b) the continuum from 5800 and $6500 \AA$, (c) for every wavelength of the Savitzki-Golay smoothed data in steps of $10 \AA$, and (d) particularly at $4950 \AA$, where the deviations are strongest (about $2 \%$ compared to $0.7 \%$ for $\lambda>5300 \AA$ ). All attempts to search for periods were applied to both LP 790-29 and the comparison star in order to eliminate apparent periods originating from the observing scheme.

In the periodograms of all four methods peaks occur in the period range between 1100 to $1400 \mathrm{~s}$. This might be due to the fact that the longer-exposed data show a very approximate repetition after $1100-1400$ s. Since no systematic variation consistent with this period exists at other wavelengths we conclude that a period between 1100 to $1400 \mathrm{~s}$ is not significant; all variations are compatible with the assumption that they are due to residual noise not entirely suppressed by the filtering process.

Finally, we folded the data with trial periods between 50 and $2500 \mathrm{~s}$ (in steps of $10 \mathrm{~s}$ ), repeated the folded spectra until they covered the total observing time interval and compared them to the original polarization data. No convincing similarities were found for any period so that we conclude that any actual short time variations should be smaller than about $\lesssim 0.7 \%$ in the continuum and $\lesssim 2 \%$ in the strongest absorption feature at $4950 \AA$.

\section{Search for secular variations}

After having found no clear indications for short term variations the question is whether secular variations over many years exist. The hypothesis that LP 790-29 has a period longer than 100 years stated by Schmidt \& Norsworthy (1991) is entirely based on two spectra taken in 1977 (Liebert et al. 1978) and broad band measurements by West (1989) taken between 1986 and 1988 . The measurement by Robert \& Moffat (1989, in the red part of the optical spectrum) seems to support this hypothesis. However, a close inspection of the data in the red continuum of LP 790-29 between 5500 and $6400 \AA$ shows slight discrepancies: a circular polarization of $10-13 \%$ in the Liebert observations, $10 \%$ in West's, and $8.2 \%$ in Robert's \& Moffat's photometry. If we add the spectro-polarimetric data by Schmidt et al. (1995) from 1994, we find that their value for the circular polarization in the red continuum (6$8 \%$ ) clearly differs from the Liebert et al. (1978) data by 4-5\%. All data from the literature are plotted in Fig. 4 together with our VLT time averaged spectro-polarimetry and ESO $3.6 \mathrm{~m}$ broad-band polarimetry by Beuermann \& Reinsch (2001). 
The scatter of the polarization near the strongest absorption feature (4500-5500 $\AA$ ) is too large to draw any definite conclusion. However, in the red continuum the star is bright and we conclude that LP 790-29 is indeed varying slowly but on a time scale smaller than previously stated. In the wavelength interval between 5500 and $6400 \AA$ the polarization changed from $11.5 \pm 1.5 \%$ in 1977 to about $8-10 \%$ in $1986 / 1987$, and decreased to $7 \pm 1 \%$ in 1994 . In 2000 , we again measured the level of the 1986/1987 polarization; thus it is possible that about half a period passed since then. If our idea is correct, the rotational period would be about 24-28 years implying that a higher degree of polarization will be reached again in 2010-2014. Long term monitoring is necessary to clarify the situation.

\section{Discussion}

Up to now, no changes of the degree of circular polarization of LP 790-29 have been reported, resulting in the conclusion that the rotational period exceeds 100 years. As an alternative to such extremely slow rotation we have tested highly time-resolved circular-polarization spectra obtained with the VLT for variations on time scales of 50 up to $2500 \mathrm{~s}$. In the extreme case of a rotational axis perpendicular to both the observer and the magnetic field axis the polarization in the continuum could vary by about $\pm 10 \%$. However, we found no evidence for rapid variations exceeding $0.7 \%$ in the continuum or $2 \%$ at the wavelengths of the absorption features. Smaller variations showing up in our observations are compatible with the assumption that they are produced by statistical noise.

The result is also compatible with fast polarimetric $R$-band photometry (5700-7400 $\AA$ ) by Beuermann \& Reinsch (2001) who found no significant periodicities between $4 \mathrm{~s}$ and 1.5 hours in their complementary study of LP 790-29 which extends to longer continuum wavelengths and looks for even shorter rotational periods.

Rapid variations cannot be completely excluded on a sub-percent level. However, since Friedrich \& Jordan (2001) also could not measure any fast variations in the case of Grw $+70^{\circ} 8247$ fast-rotation as a general scenario for magnetic white dwarfs with apparently constant flux and polarization becomes more and more improbable.

The quality of our mean circular-polarization spectrum considerably exceeds any previous data of LP 790-29. We compared it to all other measurements of circular polarization published in the literature and found that both the first data of this object taken in 1977 by
Liebert et al. (1978) and data obtained in 1994 by Schmidt et al. (1995) were discrepant from our newer data in the continuum at $\lambda>5300 \AA$. Together with broad band data by West (1989) from 1986-1988, Robert \& Moffat (1989) from 1987, and Beuermann \& Reinsch (2001) we conclude that the degree of circular polarization actually changes systematically and that there is a slight indication for a period between 24 and 28 years. Taking this period at face value the polarization at 5300-6300 $\AA$ should increase for the next 9-13 years. Since the question how much of the angular momentum is lost during stellar evolution is of crucial importance it may be worth monitoring LP 790-29 (and other magnetic white dwarfs) with large telescopes and long-exposure polarization spectra. For this purpose one high signal-to-noise circular-polarization spectrum (of one or two hours exposure time) with the VLT every year would be sufficient.

Acknowledgements. We thank the ESO staff on Cerro Paranal for observing LP 790-29 with the VLT in service mode. Work on magnetic white dwarfs in Kiel is supported by the DFG under KO-738/7-1. S. Jordan thanks K. Beuermann for financing his research in Göttingen from his DLR grant 50 OR 99036.

\section{References}

Berdyugin, A. V., \& Piirola, V. 1999, A\&A, 352, 619

Beuermann, K., \& Reinsch, K. 2001, A\&A, 381, 487

Blackman, E. G., Frank, A., Markiel, J. A., Thomas, J. H., \& Van Horn, H. M. 2001, Nature, 409, 485

Blöcker, T., \& Driebe, T. 2001, AG-Mitteilungen, 18, 98

Bues, I. 1999, in ASP Conf. Ser. 169, ed. J.-E. Solheim, \& E. G. Meištas, 240

Barstow, M. A., Jordan, S., O’Donoghue, D., et al. 1995, MNRAS, 277, 971

Burleigh, M. R., Jordan, S., \& Schweizer, W. 1999, ApJ, 510, L37

Friedrich, S., \& Jordan, S. 2001, A\&A, 367, 577

Heyl, J. S. 2000, MNRAS, 317, 310

Liebert, J., Angel, J. R. P., Stockman, H. S., \& Beaver, E. A. 1978, ApJ, 225, 181

Press, W. H., Teukolsky, S. A., Vetterling, W. T., \& Flannery, B. P. 1992, Numerical Recipes in Fortran (Cambridge Univ. Press), 644

Robert, C., \& Moffat, A. F. J. 1989, ApJ, 343, 902

Schmidt, G. D., \& Norsworthy, J. E. 1991, ApJ, 366, 270

Schmidt, G. D., Bergeron, P., \& Fegley, B. 1995, ApJ, 443, 274

Schwarzenberg-Czerny, A. 1989, MNRAS, 241, 153

Segretain, L., Chabrier, G., \& Mochkovitch, R. 1997, ApJ, 481, 355

Stellingwerf, R. F. 1978, ApJ, 224, 953

West, S. C. 1989, ApJ, 345, 511 